\title{
Use of Cryopreserved Osteogenic Matrix Cell Sheets for Bone Reconstruction
}

\author{
Tomohiko Kura ${ }^{*}$, Manabu Akahane ${ }^{2}$, Takamasa Shimizu1, Yoshinobu Uchihara1, \\ Yasuaki Tohma1, Yusuke Morita ${ }^{3}$, Munehisa Koizumi' ${ }^{1}$, Kenji Kawate ${ }^{4}$, \\ Yasuhito Tanaka1 \\ ${ }^{1}$ Department of Orthopedic Surgery, Nara Medical University School of Medicine, Kashihara, Japan \\ ${ }^{2}$ Department of Public Health, Health Management and Policy, Nara Medical University School of Medicine, \\ Kashihara, Japan \\ ${ }^{3}$ Departmentof Biomedical Engineering, Doshisha University, Kyotanabe, Japan \\ ${ }^{4}$ Department of Artificial Joint and Regenerative Medicine, Nara Medical University, Kashihara, Japan \\ Email: *tkura@naramed-u.ac.jp
}

Received 14 July 2015; accepted 5 January 2016; published 8 January 2016

Copyright @ 2016 by authors and Scientific Research Publishing Inc.

This work is licensed under the Creative Commons Attribution International License (CC BY).

http://creativecommons.org/licenses/by/4.0/

c) (i)

Open Access

\section{Abstract}

Skeletal diseases, such as nonunion and osteonecrosis, are now treatable with tissue engineering techniques. Single cell sheets called osteogenic matrix cell sheets (OMCSs) grown from cultured bone marrow-derived mesenchymal stem cells show high osteogenic potential; however, long preparation times currently limit their clinical application. Here, we report a cryopreservation OMCS transplantation method that shortens OMCS preparation time. Cryopreserved rat OMCSs were prepared using slow- and rapid-freezing methods, thawed, and subsequently injected scaffold-free into subcutaneous sites. Rapid- and slow-frozen OMCSs were also transplanted directly to the femur bone at sites of injury. Slow-freezing resulted in higher cell viability than rapid freezing, yet all two cryopreservation methods yielded OMCSs that survived and formed bone tissue. In the rapid- and slow-freezing groups, cortical gaps were repaired and bone continuity was observed within 6 weeks of OMCS transplantation. Moreover, while no significant difference was found in osteocalcin expression between the three experimental groups, the biomechanical strength of femurs treated with slow-frozen OMCSs was significantly greater than those of non-transplant at 6 weeks post-injury. Collectively, these data suggest that slow-frozen OMCSs have superior osteogenic potential and are better suited to produce a mineralized matrix and repair sites of bone injury.

\footnotetext{
"Corresponding author.
}

How to cite this paper: Kura, T., Akahane, M., Shimizu, T., Uchihara, Y., Tohma, Y., Morita, Y., Koizumi, M., Kawate, K. and Tanaka, Y. (2016) Use of Cryopreserved Osteogenic Matrix Cell Sheets for Bone Reconstruction. Stem Cell Discovery, 6, 1323. http://dx.doi.org/10.4236/scd.2016.61002 


\section{Keywords}

\section{Bone Marrow Stromal Cell, Cryopreservation, Cell Sheet, Injectable Bone, Bone Reconstruction}

\section{Introduction}

With rapid advancements in tissue engineering, various skeletal diseases and complications-such as osteonecrosis and nonunion - can be now treated using tissue-engineered bone derived from bone marrow-derived mesenchymal stem cells (BMSCs) [1]-[4]. In the fields of orthopedic and plastic surgery, open surgery for conventional bone transplantation is still the gold standard for nonunion and osteonecrosis. However, a less invasive approach for bone reconstruction is clinically preferable since it is likely to result in reduced scarring and an earlier start to rehabilitation. To accomplish this, further technical advances and investigations are required, including minimally invasive surgery using tissue engineering.

Recently, the use of cell sheets has been widely investigated as a promising technology in regenerative medicine research [5] [6]. Cell sheets have been created from the skin [7], myocardial cells [8] [9], periodontal ligaments [10], corneal epithelia [11], bladder epithelia [12], and mucosal epithelia [13]. A cell sheet transplantation technique for bone regenerative medicine was recently developed using BMSCs [14]-[16]. BMSCs are cultured in media containing dexamethasone (Dex) and ascorbic acid phosphate (AscP), and then lifted as single cell sheets using a scraper. The resulting sheets are referred to as osteogenic matrix cell sheets (OMCSs). OMCSs show high osteogenic potential in vitro and in vivo after subcutaneous scaffold-free transplantation [14] and in combination with artificial bone, such as beta-tricalcium phosphate [16]. Moreover, successful in vivo bone formation was observed after scaffold-free OMCS injection [14]. Experimental animal models have successfully demonstrated the ability for OMCSs to treat fracture nonunion [15] and ligament reconstruction [17] by enhancing bone union and callus formation between bones, as well as ligamentous surfaces and bone tunnels. Tissue invasion by injectable bone may be small, as transplantation is conducted only by injection; however, this method can be used to treat delayed union and fracture nonunion with repeated cell sheet transplantation.

One disadvantage of this technique is that the process of OMCS preparation, including BMSC culture, takes approximately 4 weeks. This may limit its clinical application. To expand the applications of OMCS transplantation in orthopedic and plastic surgery, new approaches that shorten the preparation time for cell sheets are required.

To reduce the cell preparation time required prior to transplantation, we developed a method of OMCS cryopreservation and transplantation that can be used for skeletal reconstruction. In this study, OMCSs were cryopreserved by rapid- and slow-freezing methods, thawed, and either injected scaffold-free as injectable bone to a subcutaneous site or transplanted directly onto sites of bone defects in rat femurs. The capacity of grafts to repair bone was then monitored over the course of 6 weeks.

\section{Methods}

\subsection{Ethics Statement}

The care and handling of the rats used in this study were approved by our institute's Animal Care Committee, and met the standards of the National Institutes of Health. Male Fischer 344 (F344) rats were purchased from Japan SLC (Shizuoka, Japan), and used as donors and recipients.

\subsection{Bone Marrow Cell Preparation}

Bone marrow cell preparation was conducted according to previous reports [14]-[18]. Briefly, bone marrow cells were obtained from the femur shafts of 7-week-old male F344 rats. Both femur ends were cut and the bone marrow was flushed out using $10 \mathrm{~mL}$ of standard culture medium [minimal essential medium] (Nacalai Tesque Inc.; Kyoto, Japan) with 15\% fetal bovine serum (Gibco Life Technologies; Carlsbad, CA, USA) and antibiotics (100 U/mL penicillin and $100 \mu \mathrm{g} / \mathrm{mL}$ streptomycin; Nacalai Tesque Inc.). Released cells were collected into two T-75 flasks (BD Falcon; BD Biosciences; Franklin Lakes, NJ, USA) containing $15 \mathrm{~mL}$ of standard culture medium. Cells were cultured in an incubator under $5 \% \mathrm{CO}_{2}$ at $37^{\circ} \mathrm{C}$. After reaching confluence, primary cultured 
cells were trypsinized from T-75 flasks using trypsin/ethylenediaminetetraacetic acid (Nacalai Tesque Inc.).

\subsection{OMCS Preparation}

OMCSs were prepared according to previously reported methods [14]-[18]. Briefly, primary cultured BMSCs were seeded at $1 \times 10^{4}$ cells $/ \mathrm{cm}^{2}$ in 10 -cm dishes $(100 \times 20 \mathrm{~mm}$; BD Falcon) containing $10 \mathrm{nM}$ Dex (Sigma; St. Louis, MO, USA) and AscP (L-ascorbic acid phosphate magnesium salt n-hydrate, $82 \mu \mathrm{g} / \mathrm{mL}$; Wako Pure Chemical Industries; Kyoto, Japan), and grown until confluent (approximately 14 days). Cells were rinsed twice with phosphate-buffered saline (PBS; Gibco) and then OMCSs were lifted using a scraper.

\subsection{Cryopreserved OMCS Preparation}

Cryopreserved OMCSs were prepared using rapid- and slow-freezing methods. For the rapid- and slow-freezing groups, tweezers were used to transfer OMCSs grown in 10-cm dishes to 2-mL cryovials (cryogenic vial; BD Falcon) containing $500 \mu \mathrm{L}$ cryopreservation medium (Cell Banker 1; Juji Field, Inc.; Tokyo, Japan).The OMCSs in the slow-freezing group were then cryopreserved in a controlled-rate freezing chamber (Bicell; Nihon Freezer, Tokyo, Japan) placed in a $-80^{\circ} \mathrm{C}$ freezer. The rate of cryopreservation was set at $-1^{\circ} \mathrm{C} / \mathrm{min}$ from $+4^{\circ} \mathrm{C}$ to $-80^{\circ} \mathrm{C}$. After overnight storage at $-80^{\circ} \mathrm{C}$, OMCSs were stored in a liquid nitrogen tank. For the rapid-freezing group, cryovials were placed directly into a $-80^{\circ} \mathrm{C}$ freezer with no controlled-rate freezing chamber. After overnight storage at $-80^{\circ} \mathrm{C}$, cryovials were transferred and stored in a liquid nitrogen tank. In both groups, temperature changes in the cryopreservation medium were measured in cryovials using a thermometer sensor (CENTER370 RTD thermometer; Center Technology Group; New Taipei, Taiwan),which was inserted into the cryopreservation medium through a hole in the cryovial cap.

After cryopreservation, samples were placed in a water bath at $37^{\circ} \mathrm{C}$ until completely thawed. Thawed OMCSs were rinsed twice with PBS prior to use in subsequent experiments.

\subsection{Cell Viability Assay}

Viability of OMCSs in both the rapid- and slow-freezing groups was determined using a previously reported method based on tetrazolium reductase activity (Cell Counting Kit-8 [WST-8]; Dojindo; Kumamoto, Japan) [18] [19]. Briefly, standard curves were generated using OMCSs cultured in 6-, 12-, 24-, and 48-well culture plates (BD Falcon; $\mathrm{n}=5$ per plate). The differently sized OMCSs were harvested from each culture plate using a scraper, and incubated in $1 \mathrm{~mL}$ culture medium in a 95\% humidified atmosphere with $5 \% \mathrm{CO}_{2}$ at $37^{\circ} \mathrm{C}$ for $24 \mathrm{~h}$. Next, samples were placed in WST-8 solution ( $100 \mu \mathrm{L}$ in $1 \mathrm{~mL}$ culture medium) in culture wells. After $2 \mathrm{~h}$ incubation, the solution obtained from each culture well was analyzed using a spectrophotometer set at $450 \mathrm{~nm}$. A linear relationship (correlation $\mathrm{R}^{2}=0.969$ ) was observed between the average optical density and number of seeded cells per unit volume of cultured medium (cells/mL).

OMCSs cultured in 12-well plates $(n=5)$ were cryopreserved using the same protocol for the rapid- and slow-freezing groups, and after thawing at $37^{\circ} \mathrm{C}$ were incubated in the same manner as for standard curve preparation. Using the standard curve, the number of viable cells in OMCSs from each group was analyzed before freezing and after thawing. Measurement of cell viability was the same as for standardization. For all samples, cell viability was calculated at $24 \mathrm{~h}$ after initiation of thawing as a percentage relative to the fresh group.

\subsection{Osteogenesis of Injected Cryopreserved OMCSs at Ectopic Sites}

Osteogenesis of rapid- and slow-freezing OMCSs was compared to that of fresh OMCSs. OMCSs obtained from anentire 10-cm dish were collected into $500 \mu \mathrm{L}$ standard culture medium in a 1-mL syringe (JMS Co. Ltd.; Tokyo, Japan), and then injected into a subcutaneous site on the backs of F344 rats $(n=12)$ using a 16-G needle (Terumo; Tokyo, Japan). Thawed OMCSs were transplanted immediately without culturing. Four weeks after injection, all samples were harvested and fixed in 10\% neutral buffer formalin for 2 days. X-ray images of each sample were then obtained. Next, samples were decalcified in K-CX solution (Falma Co.; Tokyo, Japan), embedded in paraffin, and cut in parallel down the middle prior to hematoxylin and eosin (H\&E) and Sirius red staining. 


\subsection{RNA Isolation and Real-Time Quantitative Polymerase Chain Reaction (PCR)}

To confirm osteogenesis in the harvested OMCSs, levels of osteocalcingene expression was measured. RNA was isolated from five samples from each group using an Isogen RNA extraction kit (Nippon Gene Co. Ltd.; Toyama, Japan). Harvested samples were placed in matrix bead vials with Isogen solution and disrupted using a Fast Prep FP120 cell disrupter (Qbiogene, Inc.; Carlsbad, CA, USA). The remaining steps of RNA isolation were performed according to the manufacturer's instructions.

To measure mRNA expression levels, real-time quantitative PCR (ABI PRISM 7700 Sequence Detection System; Applied Biosystems; Norwalk, CT, USA) was performed using appropriate primers and specific fluorogenic probes for rat cDNA sequences of osteocalcin and glyceraldehyde-3-phosphate dehydrogenase (GAPDH), as described previously [14] [20] [21]. Target osteocalcin mRNA levels in injected OMCS samples collected from each group $(n=5)$ were compared after correcting to GAPDH levels. We used GAPDH as an internal standard to adjust for sample differences in reverse transcription efficiency. Osteocalcin (Rn01455285 g1), GAPDH (Rn99999916 s1) primer and probe sets were purchased from Applied Biosystems (Foster City, CA, USA). Thermal cycling conditions were $10 \mathrm{~min}$ at $95^{\circ} \mathrm{C}$ for activation of universal mixture Ampli Taq Gold Polymerase, followed by 35 cycles of $15 \mathrm{~s}$ at $95^{\circ} \mathrm{C}$ for denaturing and $1 \mathrm{~min}$ at $60^{\circ} \mathrm{C}$ for annealing and extension. PCR experiments were conducted in duplicate.

\subsection{OMCS Osteogenesis at Bone Defect Sites}

An experimental rat femur bone defect model was generated under isoflurane anesthesia. Briefly, a lateral incision was made on the hind limb, and the vastus muscle was divided longitudinally to expose the right femur. After making a triangle osteotomy of the femoral shaft using an oscillating mini saw, a 1.2-mm K-wire was inserted into the femoral shaft intramedullary from the distal femoral condyle in a retrograde fashion, resulting in rigid fixation to maintain the bone defect. The femur bone defect was wrapped using two pieces of OMCSs from the rapid- and slow-freezing groups. In the non-transplant group, the right femur was treated in the same manner but without OMCS transplantation. Unprotected weight bearing was allowed immediately after the operation.

Post-operative femur X-ray photographs were taken under anesthesia at 3 and 6 weeks to evaluate callus and bridging bone formation at the bone defect site. Two femurs from each group were harvested at 3 and 6 weeks post-transplant. After removing the intramedullary pins from the femur, the surrounding muscle was dissected, and harvested femurs were fixed in $10 \%$ neutral buffer formalin, decalcified using ethylene diamine tetraacetic acid solution, and embedded in paraffin. Femurs were cut longitudinally, H\&E stained, and histologically evaluated.

\subsection{Biomechanical Analysis}

Fifteen rats were used for biomechanical evaluation. Femurs were harvested at 6 weeks post-operatively, and three-point bending tests were performed in a vertical direction using a universal testing machine (EZgraph, Shimadzu; Kyoto, Japan). After removing the intramedullary pins, the harvested femurs were fixed in a clamping jig. All 15 femurs (five from each group) were bent at a constant rate of $10 \mathrm{~mm} / \mathrm{min}$, and the maximum force at failure was recorded.

\subsection{Statistical Analysis}

Cell viability, real-time PCR, and biomechanical test values are represented by means \pm standard deviation. Multiple comparisons among groups were evaluated by one-way analysis of variance with post-hoc multiple comparisons using Tukey's test. $P<0.05$ was considered statistically significant. All statistical analysis were conducted by using Ekuseru Toukei 2010 (Social Survey Research Information Co., Ltd; Tokyo Japan).

\section{Results}

\subsection{Temperature Change during Cryopreservation}

Representative temperature changes in the rapid- and slow-freezing groups are shown in Figure 1. In the slow-freezing group, almost $3 \mathrm{~h}$ was required for cryopreservation from $0^{\circ} \mathrm{C}$ to $-80^{\circ} \mathrm{C}$. In contrast, in the rapid- 


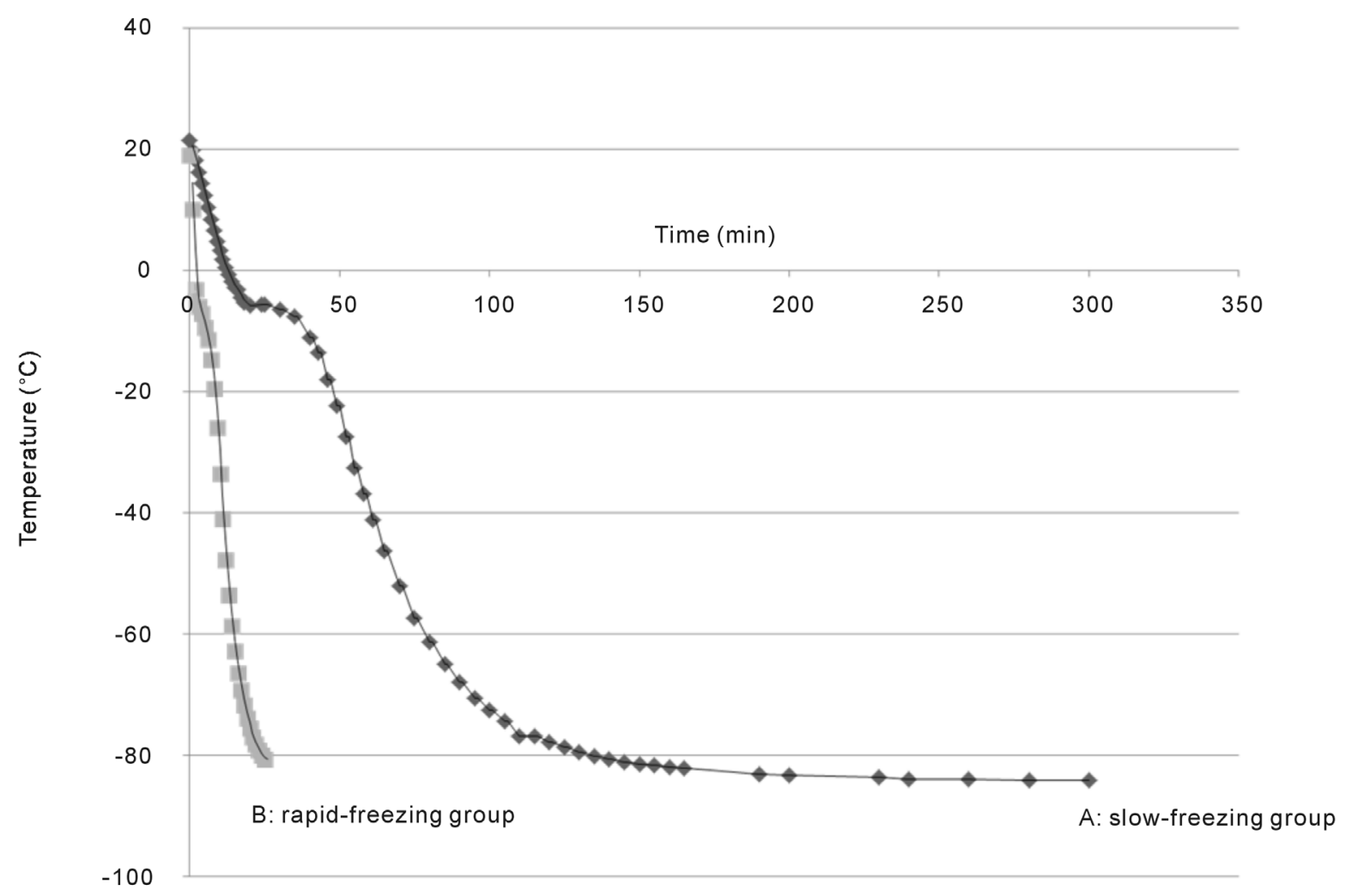

Figure 1. Temperature change during cryopreservation of osteogenic matrix cell sheets by slow-freezing (A) and rapidfreezing (B). A sensor was inserted into the cryopreservation medium through a hole in the cryovial cap. Measurements $(\mathrm{n}=$ 1) were repeated three times, with similar results.

freezing group, only 25 min was needed for cryopreservation from $0^{\circ} \mathrm{C}$ to $-80^{\circ} \mathrm{C}$. Measurements $(\mathrm{n}=1)$ were repeated three times, with all recordings showing a similar trend.

\subsection{Cell Viability of OMCSs}

The cell viability of cryopreserved and thawed OMCSs is shown in Figure 2. There was no significant difference in cell viability between the fresh and slow-freezing groups, whereas cell viability in the rapid-freezing group was significantly lower than that of the fresh group. Average cell viability in the slow-freezing group was approximately $70 \%$ that of the fresh group.

\subsection{Osteogenesis of Injected Cryopreserved OMCSs at Ectopic Sites}

The macroscopic appearances of harvested specimens obtained from the fresh, rapid-, slow-freezing groups are shown in Figure 3(A), Figure 3(G) and Figure 3(M). Specimens appeared as hard masses and showed calcification, as determined by X-ray photography (Figure 3(B), Figure 3(H), Figure 3(N)).H\&E staining showed a bone matrix with osteocytes in each groups (Figure 3(C), Figure 3(D), Figure 3(I), Figure 3(J), Figure 3(O), Figure $3(\mathrm{P})$ ). Sirius red staining was used to assess the extent of collagen deposition (predominantly collagen type I and III fibers), and positive staining was observed on newly formed bone (Figure 3(E), Figure 3(F), Figure 3(K), Figure 3(L), Figure 3(Q), Figure 3(R)). These observations indicate that after injection, OMCSs in the fresh, rapid-freezing, and slow-freezing groups were viable in subcutaneous sites and form bone tissue in a scaffold-free manner.

Osteocalcin expression levels evaluated by real-time PCR are shown in Figure 4. Osteocalcin expression level was $0.97 \pm 0.44,1.33 \pm 0.57$ and $1.08 \pm 0.34$ in fresh, rapid-freezing and slow-freezing groups, respectively. There was no significant difference in osteocalcin expression among the fresh, rapid-freezing, and slow-freezing groups. 


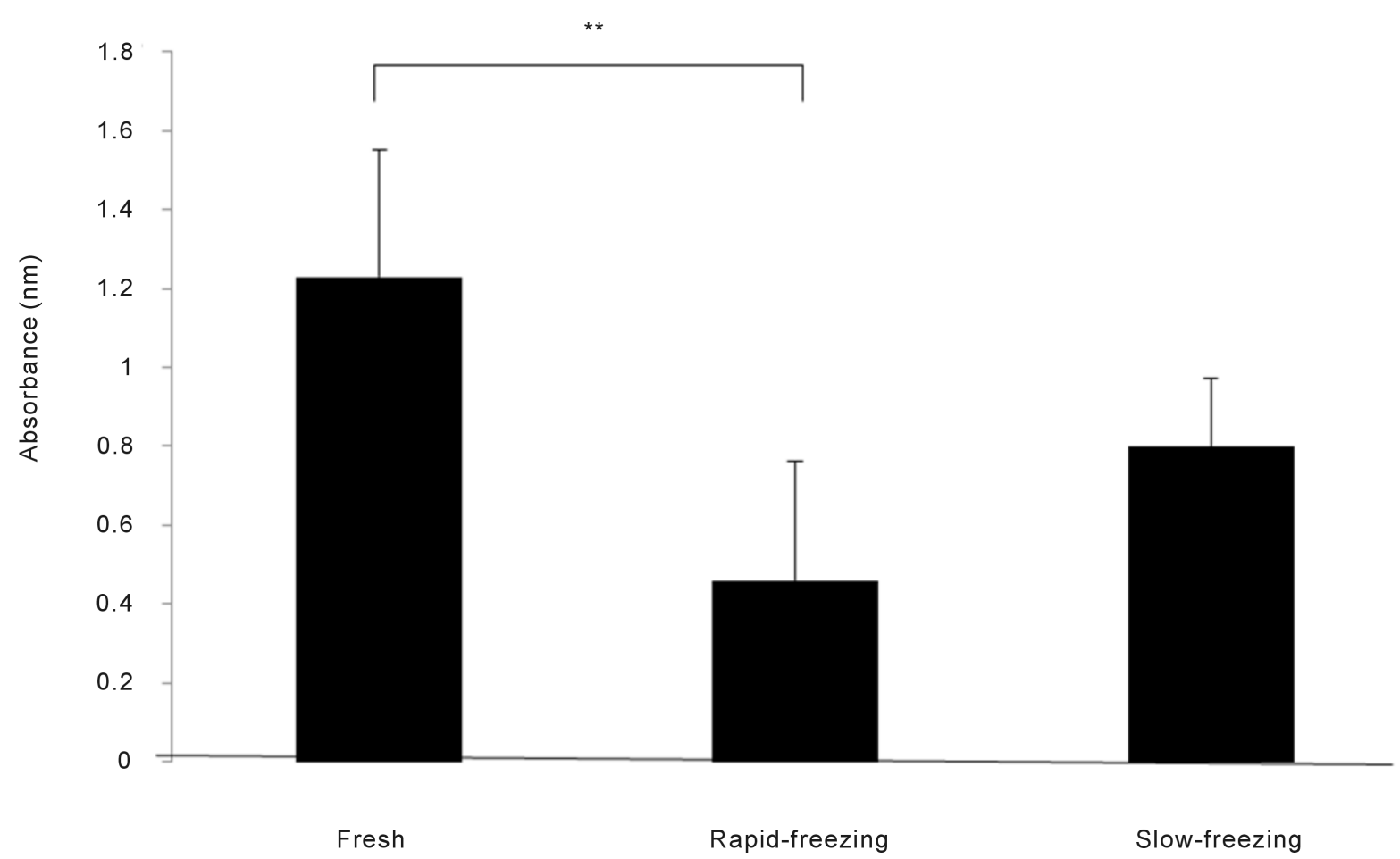

Figure 2. Cell viability in fresh, slow-, and rapid-frozen osteogenic matrix cell sheets. Viability was measured using tetrazolium activity, and compared to standard curves $(n=5)$. Cell viability differed significantly between the fresh and rapidfreezing groups. ${ }^{*} P<0.05 .{ }^{* *} P<0.01$.

\subsection{Osteogenesis of OMCSs at Bone Defect Sites}

In the rapid- and slow-freezing groups, radio graphs taken 3 weeks after transplantation of cryopreserved/thawed OMCSs showed callus formation around bone defect sites (Figure 5(A)). The cortical gap at the defect site disappeared by 6 weeks post-transplantation. In the non-transplant group, only faint callus formation was seen at 3 weeks, and the cortical gap was still present even at 6 weeks. At 6 weeks, cortical bone continuity was observed in the rapid- and slow-freezing group but not in the non-transplant group (Figure 5(B)).

The maximum forces applied to femurs in the non-transplant, rapid-, and slow-freezing groups are shown in Figure 6. While no significant difference in the maximum force at defect sites was observed between the nontransplant and rapid-freezing groups at 6 weeks, femurs from the slow-freezing group were able to withstand much greater force than those from the non-transplant group.

\section{Discussion}

The results of our study demonstrate that cryopreserved OMCSs have osteogenesis. Rapid freezing decreased OMCS cell viability to a greater degree compared to slow freezing. Following injection of rapid- and slow-frozen OMCSs into subcutaneous sites or transplantation to bone defect sites, we observed abundant bone formation or bone union. Osteocalcin was used as a marker of bone maturation, since its expression increases continuously throughout this process; however, no significant difference in osteocalcin expression among three groups. Moreover, in femurs transplanted with slow-frozen OMCSs, our biomechanical examination demonstrated significantly higher bone strength against bending stress than non-transplant group, indicating that slow-frozen OMCSs are useful for skeletal reconstruction. Our group has previously reported on the usefulness of fresh sheets [15]. In cases of delayed union, fracture bone formation is generally promoted using low-intensity pulsed ultrasound [22] [23] or pulsed electromagnetic fields [24]. If the fracture develops into a nonunion, an invasive operation, such as bone transplantation with autologous (including vascularized) bone grafts, is commonly performed. However, such procedures require sacrificing intact bone such as that of the pelvis or fibula, and there is limited bone available for autologous transplantation. Therefore, a less invasive technique is required. 


\section{Fresh}
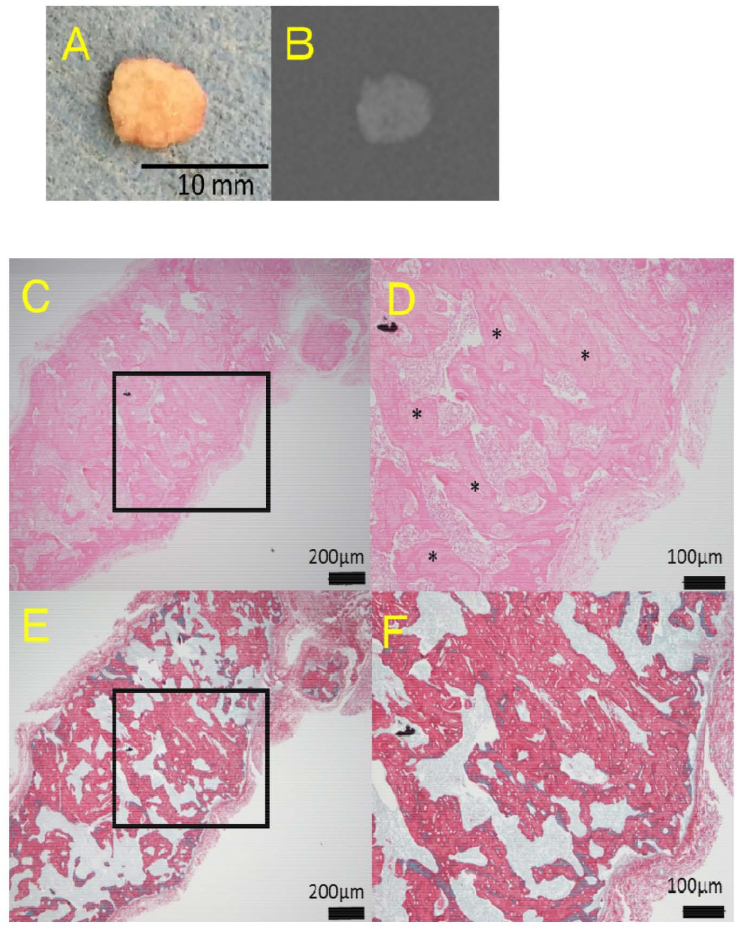

Rapid-freezing
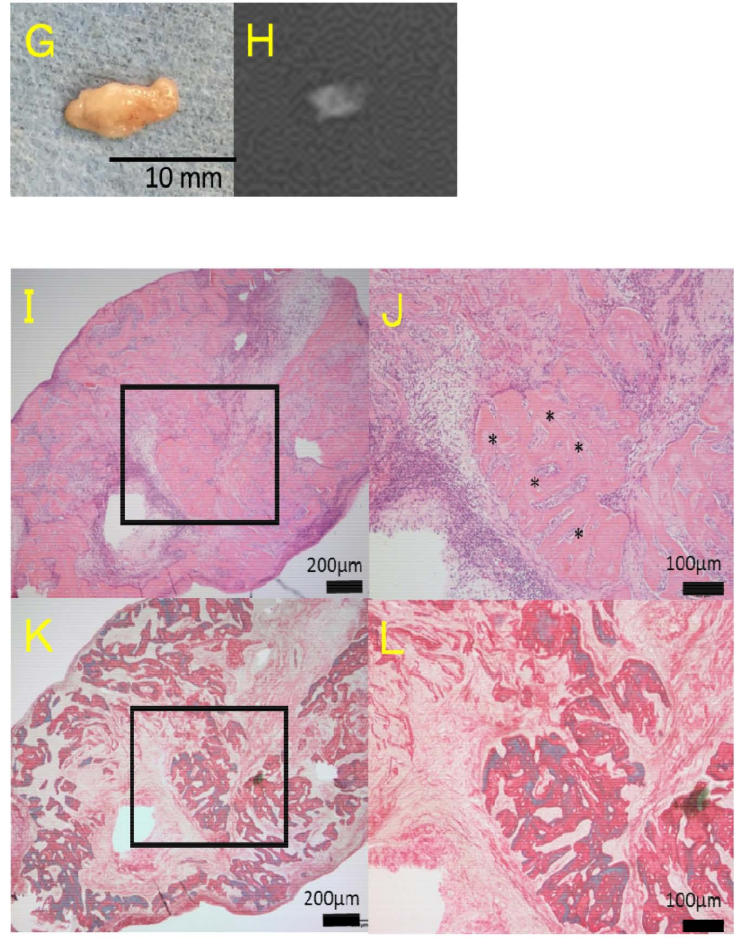

\section{Slow-freezing}
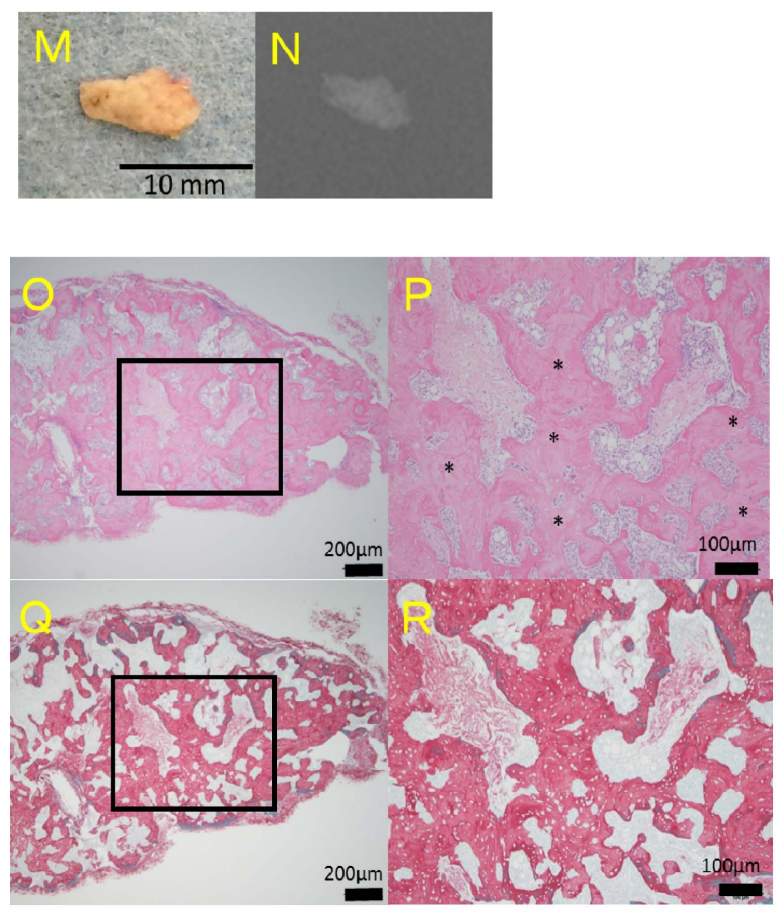

Figure 3. Osteogenesis after subcutaneous injection of fresh (A)-(F), rapid-frozen (G)-(L), and slow-frozen (M)-(R) osteogenic matrix cell sheets. (A), (G), (M): macroscopic appearance. (B), (H), (N): X-ray photography. (C), (D), (I), (J), (O), (P): hematoxylin and eosinstaining. Asterisks indicate newly formed bone. (E), (F), (K), (L), (Q), (R): Sirius red staining. (D), (F), (J), (L), (P), (R): high-magnification images of each rectangular area (C)-(D), (E)-(F), (I)-(J), (K)-(L), (O)-(P), (Q)-(R). 


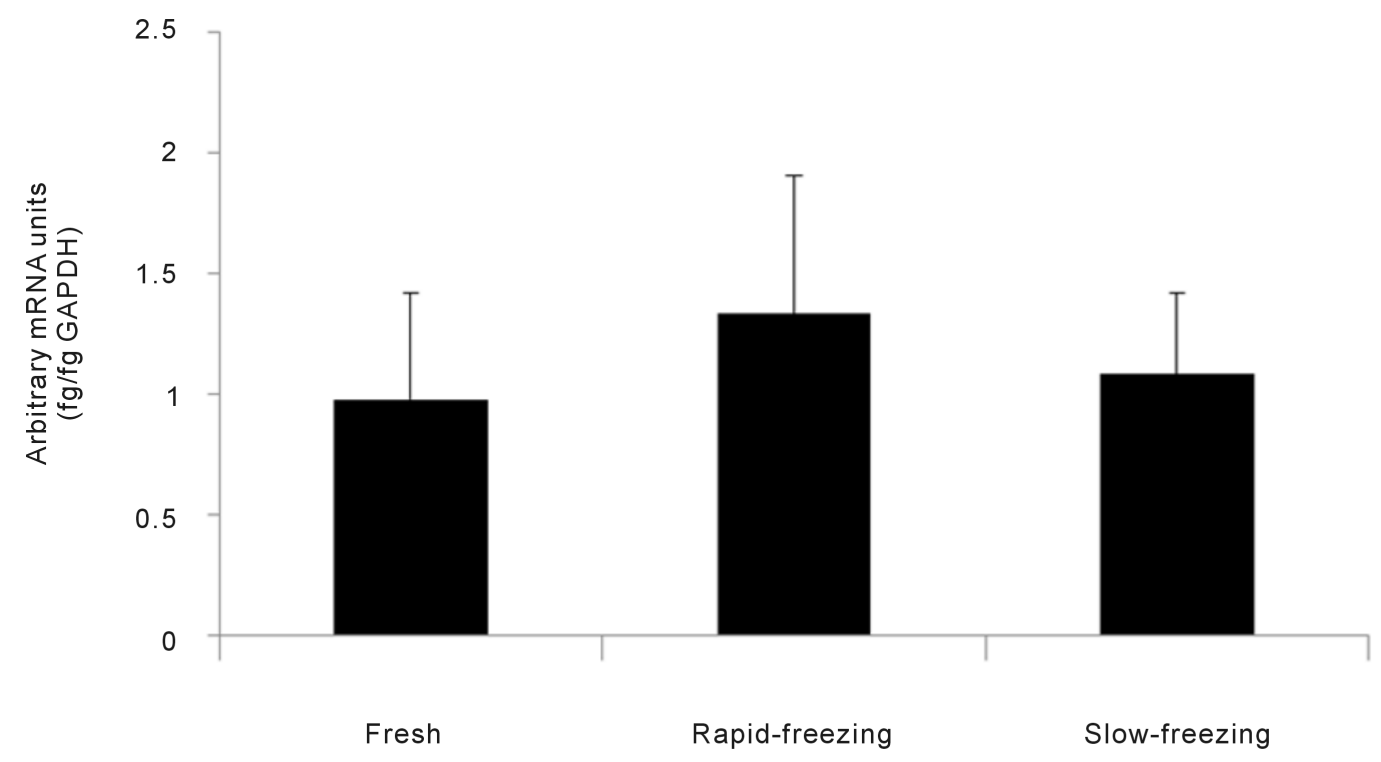

\section{Osteocalcin}

Figure 4. Osteocalcin mRNA expression after subcutaneous injection of the fresh, rapid-, and slow-freezing groups. There was no significant difference in osteocalcin expression. $n=5$; ${ }^{*} P<0.05$.

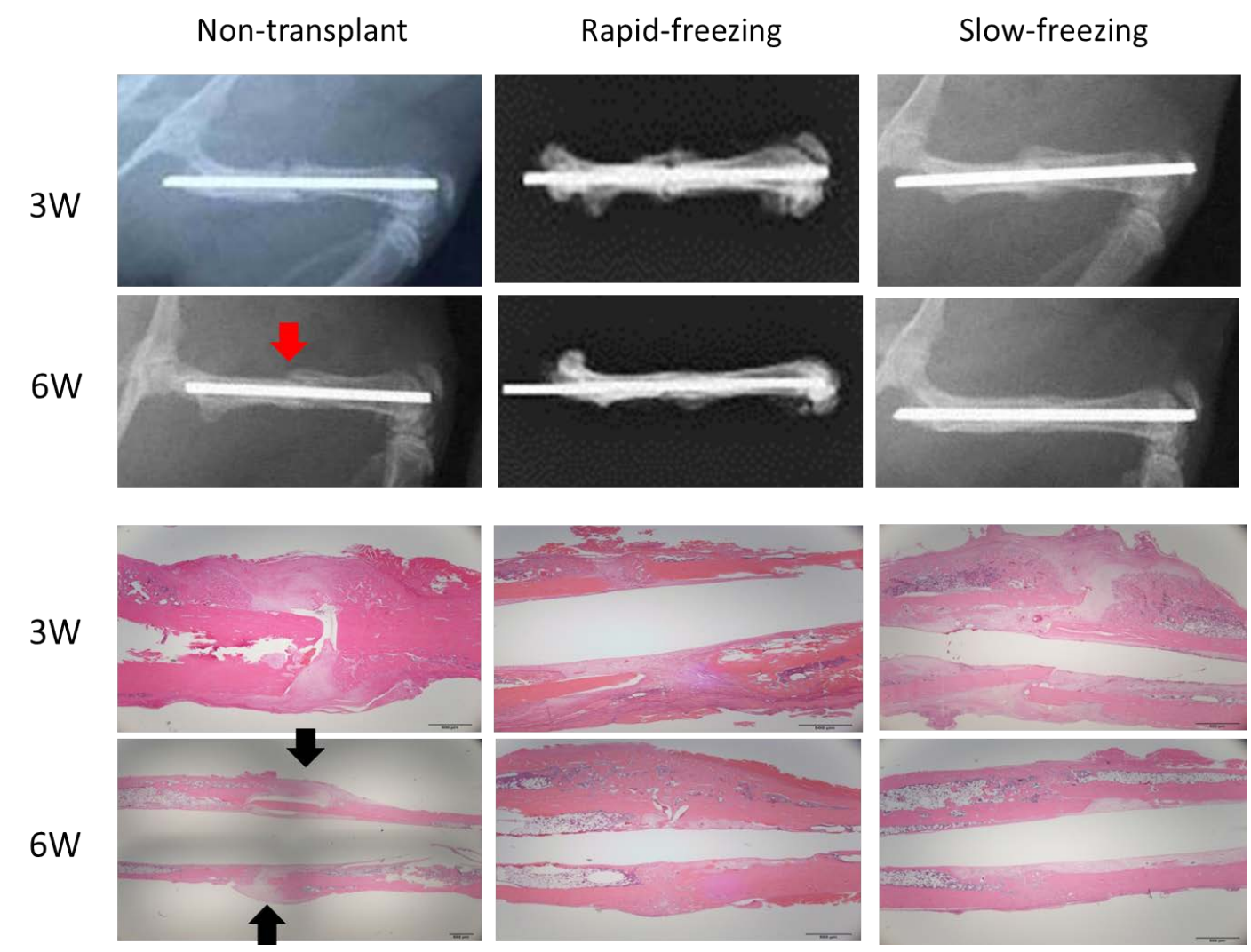

Figure 5. Osteogenesis at bone defect sites after injection with rapid- or slow-frozen osteogenic matrix cell sheets as compared to non-transplant. A: radiographs at 3 and 6 weeks $(3 \mathrm{~W}, 6 \mathrm{~W})$. B: representative histological sections at 3 and 6 weeks. Red arrow, cortical gap; black arrows, soft tissue. 


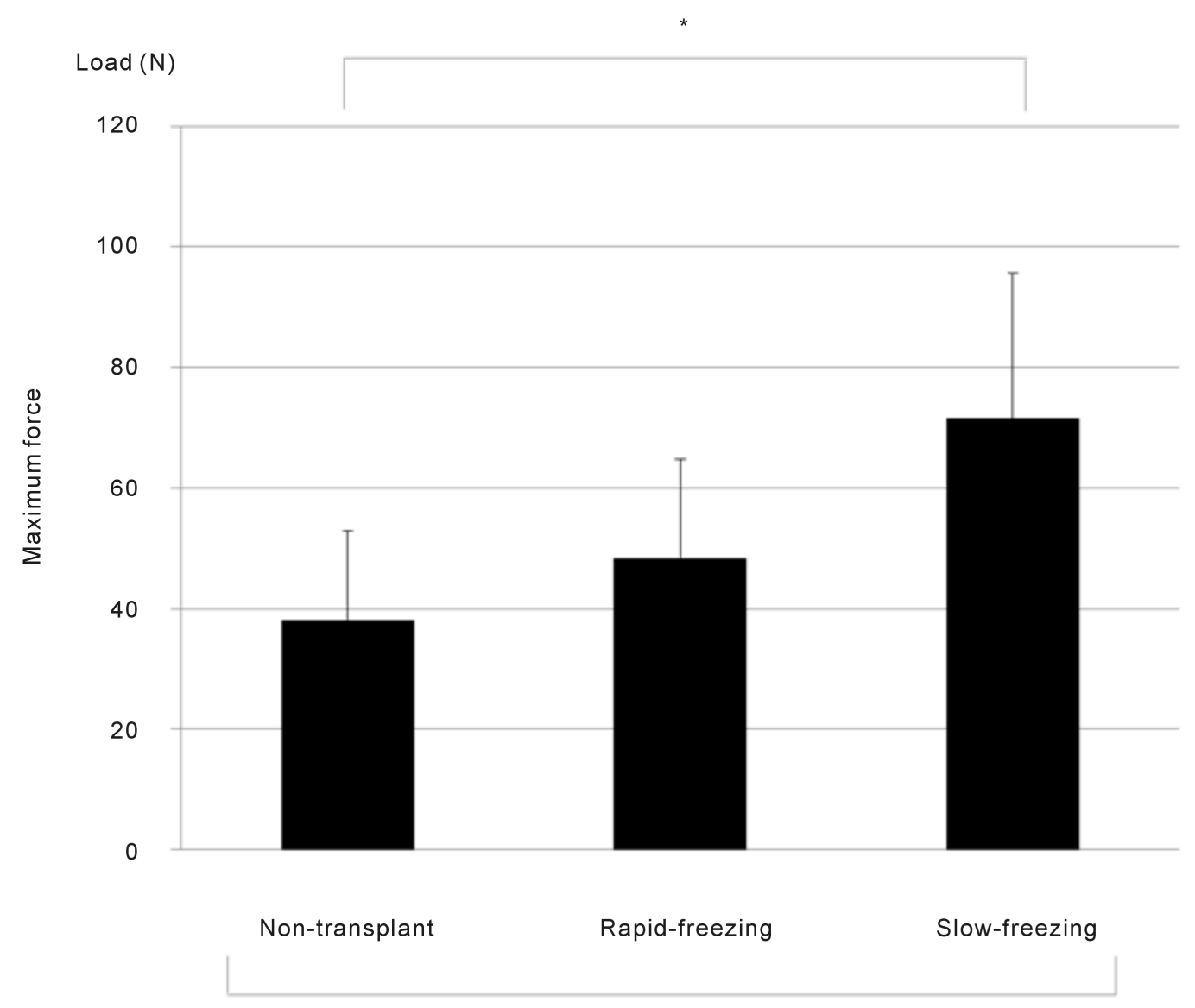

$6 \mathrm{~W}$

Figure 6. Biomechanical analysis. Results of three-point bending tests of femurs at 6-weeks post-transplant (n $=5$ per group). ${ }^{*} P<0.05$.

In the orthopedic and plastic surgery fields, distraction osteogenesis techniques are commonly applied to the lower extremities and craniofacial skeleton. Robiony et al. [25] and Kitoh et al. [26] reported that distraction can be accelerated by mesenchymal stem cells and platelet-rich plasma transplantation, resulting in a shortened treatment period. Our results suggest that injectable bone using slow-frozen OMCSs may also enhance or speed up bone formation in cases of delayed union and distraction osteogenesis, and would enable repeated treatments.

Successful clinical application of regenerative treatment for skeletal reconstruction based on cell transplantation requires precise timing, so that cell preparation coincides with the time of the operation. Decreasing cell preparation time is currently an unmet clinical need. Although prior BMSC cryopreservation is a technique that may shorten the process, cultivation time is nonetheless required before transplantation to prepare the cell/scaffold constructs for thawed BMSCs [27]. An OMCS cryopreservation method was developed to overcome this disadvantage [18]. The previously reported cryopreservation method used a rapid-freezing technique that resulted in deterioration of OMCS cell viability during the cryopreservation/thawing process. Here we have demonstrated that a slow-freezing method for cryopreserved OMCSs maintains cell viability and promotes osteogenesis. Both nonunion and distraction bone reconstructions tend to require long treatment periods; thus, OMCS cryopreservation will enable the rapid preparation and immediate use of cell sheets that may expedite this process.

Our study had a few limitations. First, we used rat BMSCs, and further experiments using human BMSCs must be performed to evaluate clinical applications of this technique. Second, the experimental bone defect we investigated was relatively small, and further study using a critical-sized bone defect model is needed. Third, we showed bone formation after cryopreserved OMCS injection to subcutaneous sites; however, it is also necessary to examine the bone union capacity of OMCSs at defect sites to fully evaluate the effects of repeated OMCS in- 
jection. Thus, additional research is needed to clearly determine the clinical significance of cryopreserved OMCSs, and we are preparing further studies to address these points.

\section{Conclusion}

Cryopreserved OMCSs prepared using slow-freezing and rapid-thawing methods have osteogenic potential. Cryopreserved/thawed OMCSs are capable of producing a mineralized matrix at bone defect sites that results in bone union. Slow-frozen OMCSs are useful for skeletal reconstruction such as in cases of bone defects, nonunion, and osteonecrosis. This method shows good potential for reducing the cell preparation time needed for OMCS treatment.

\section{Acknowledgements}

We thank F. Kunda, M. Yoshimura, and M. Matsumura (Nara Medical University School of Medicine, Japan) for technical assistance. Y. Katoh and T. Inoue (Department of Biomedical Engineering, Doshisha University) for biomechanical analysis. This study was conducted with the support of a Grant-in-Aid for Young Scientists.

\section{Competing Interests}

The authors declare that there is no conflict of interest regarding the publication of this paper.

\section{References}

[1] Hernigou, P. and Beaujean, F. (2002) Treatment of Osteonecrosis with Autologous Bone Marrow Grafting. Clinical Orthopaedics and Related Research, 405, 14-23. http://dx.doi.org/10.1097/00003086-200212000-00003

[2] Morishita, T., Honoki, K., Ohgushi, H., Kotobuki, N., Matsushima, A. and Takakura, Y.(2006) Tissue Engineering Approach to the Treatment of Bone Tumors: Three Cases of Cultured Bone Grafts Derived from Patients' Mesenchymal Stem Cells. Artificial Organs, 30, 115-118. http://dx.doi.org/10.1111/j.1525-1594.2006.00190.x

[3] Quarto, R., Mastrogiacomo, M., Cancedda, R., Kutepov, S.M., Mukhachev, V., Lavroukov, A., Kon, E. and Marcacci, M. (2001) Repair of Large Bone Defects with the Use of Autologous Bone Marrow Stromal Cells. New England Journal of Medicine, 344, 385-386. http://dx.doi.org/10.1056/NEJM200102013440516

[4] Oryan, A., Alidadi, S., Moshiri, A. and Maffulli, N. (2014) Bone Regenerative Medicine: Classic Options, Novel Strategies, and Future Directions. Journal of Orthopaedic Surgery and Research, 9, 18.

http://dx.doi.org/10.1186/1749-799X-9-18

[5] Yamato, M. and Okano, T. (2004) Cell Sheet Engineering. Materials Today, 7, 42-47. http://dx.doi.org/10.1016/S1369-7021(04)00234-2

[6] Elloumi-Hannachi, I., Yamato, M. and Okano, T. (2010) Cell Sheet Engineering: A Unique Nanotechnology for Scaffold-Free Tissue Reconstruction with Clinical Applications in Regenerative Medicine. Journal of Internal Medicine, 267, 54-70. http://dx.doi.org/10.1111/j.1365-2796.2009.02185.x

[7] Yamato, M., Utsumi, M., Kushida, A., Konno, C., Kikuchi, A. and Okano, T. (2001) Thermo-Responsive Culture Dishes Allow the Intact Harvest of Multilayered Keratinocyte Sheets without Dispase by Reducing Temperature. Tissue Engineering, 7, 473-480. http://dx.doi.org/10.1089/10763270152436517

[8] Shimizu, T., Sekine, H., Isoi, Y., Yamato, M., Kikuchi, A. and Okano, T. (2006) Long-Term Survival and Growth of Pulsatile Myocardial Tissue Grafts Engineered By the Layering of Cardiomyocyte Sheets. Tissue Engineering, 12, 499-507. http://dx.doi.org/10.1089/ten.2006.12.499

[9] Shimizu, T., Yamato, M., Kikuchi, A. and Okano, T. (2001) Two-Dimensional Manipulation of Cardiac Myocyte Sheets Utilizing Temperature-Responsive Culture Dishes Augments the Pulsatile Amplitude. Tissue Engineering, 7, 141-151. http://dx.doi.org/10.1089/107632701300062732

[10] Akizuki, T., Oda, S., Komaki, M., Tsuchioka, H., Kawakatsu, N., Kikuchi, A., Yamato, M., Okano, T. and Ishikawa, I. (2005) Application of Periodontal Ligament Cell Sheet for Periodontal Regeneration: A Pilot Study in Beagle Dogs. Journal of Periodontal Research, 40, 245-251. http://dx.doi.org/10.1111/j.1600-0765.2005.00799.x

[11] Nishida, K. (2003) Tissue Engineering of the Cornea. Cornea, 22, S28-S34. http://dx.doi.org/10.1097/00003226-200310001-00005

[12] Shiroyanagi, Y., Yamato, M., Yamazaki, Y., Toma, H. and Okano, T. (2004) Urothelium Regeneration Using Viable Cultured Urothelial Cell Sheets Grafted on Demucosalized Gastric Flaps. British Journal of Urology International, 93, 1069-1075. http://dx.doi.org/10.1111/j.1464-410X.2004.04783.x 
[13] Ohki, T., Yamato, M., Murakami, D., Takagi, R., Yang, J., Namiki, H., Okano, T. and Takasaki, K. (2006) Treatment of Oesophageal Ulcerations Using Endoscopic Translation of Tissue-Engineered Autologous Oral Mucosal Epithelial Cell Sheets in a Canine Model. Gut, 55, 1704-1710. http://dx.doi.org/10.1136/gut.2005.088518

[14] Akahane, M., Shigematsu, H., Tadokoro, M., Ueha, T., Matsumoto, T., Tohma, Y., Kido, A., Imamura, T. and Tanaka, Y. (2010) Scaffold-Free Cell Sheet Injection Results in Bone Formation. Journal of Tissue Engineering and Regenerative Medicine, 4, 404-411. http://dx.doi.org/10.1002/term.259

[15] Nakamura, A., Akahane, M., Shigematsu, H., Tadokoro, M., Morita, Y., Ohgushi, H., Dohi, Y., Imamura, T. and Tanaka, Y. (2010) Cell Sheet Transplantation of Cultured Mesenchymal Stem Cells Enhances Bone Formation in a Rat Nonunion Model. Bone, 46, 418-424. http://dx.doi.org/10.1016/j.bone.2009.08.048

[16] Akahane, M., Ueha, T., Shimizu, T., Shigematsu, H., Kido, A., Omokawa, S., Kawate, K., Imamura, T. and Tanaka, Y. (2010) Cell Sheet Injection as a Technique of Osteogenic Supply. International Journal of Stem Cells, 3, 138-143. http://dx.doi.org/10.15283/ijsc.2010.3.2.138

[17] Inagaki, Y., Uematsu, K., Akahane, M., Morita, Y., Ogawa, M., Ueha, T., Shimizu, T., Kura, T., Kawate, K. and Tanaka, Y. (2013) Osteogenic Matrix Cell Sheet Transplantation Enhances Early Tendon Graft to Bone Tunnel Healing in Rabbits. BioMed Research International, 2013, Article ID: 842192. http://dx.doi.org/10.1155/2013/842192

[18] Shimizu, T., Akahane, M., Ueha, T., Kido, A., Omokawa, S., Kobata, Y., Murata, K., Kawate, K. and Tanaka, Y. (2013) Osteogenesis of Cryopreserved Osteogenic Matrix Cell Sheets. Cryobiology, 66, 326-332. http://dx.doi.org/10.1016/j.cryobiol.2013.03.011

[19] Kito, K., Kagami, H., Kobayashi, C., Ueda, M. and Terasaki, H. (2005) Effects of Cryopreservation on Histology and Viability of Cultured Corneal Epithelial Cell Sheets in Rabbit. Cornea, 24, 735-741. http://dx.doi.org/10.1097/01.ico.0000154405.68536.a4

[20] Shimaoka, H., Dohi, Y., Ohgushi, H., Ikeuchi, M., Okamoto, M., Kudo, A., Kirita, T. and Yonemasu, K. (2004) Recombinant Growth/Differentiation Factor-5 (GDF-5) Stimulates Osteogenic Differentiation of Marrow Mesenchymal Stem Cells in Porous Hydroxyapatite Ceramic. Journal of Biomedical Materials Research A, 68, 168-176. http://dx.doi.org/10.1002/jbm.a.20059

[21] Tohma, Y., Ohgushi, H., Morishita, T., Dohi, Y., Tadokoro, M., Tanaka, Y. and Takakura, Y. (2008) Bone MarrowDerived Mesenchymal Cells Can Rescue Osteogenic Capacity of Devitalized Autologous Bone. Journal of Tissue Engineering and Regenerative Medicine, 2, 61-68. http://dx.doi.org/10.1002/term.67

[22] Rubin, C., Bolander, M., Ryaby, J.P. and Hadjiargyrou, M. (2001) The Use of Low-Intensity Ultrasound to Accelerate the Healing of Fractures. Journal of Bone and Joint Surgery, 83, 259-270.

[23] Heckman, J.D., Ryaby, J.P., McCabe, J., Frey, J.J. and Kilcoyne, R.F. (1994) Acceleration of Tibial Fracture-Healing by Non-Invasive, Low-Intensity Pulsed Ultrasound. Journal of Bone and Joint Surgery, 76, 26-34.

[24] Assiotis, A., Sachinis, N.P. and Chalidis, B.E. (2012) Pulsed Electromagnetic Fields for the Treatment of Tibial Delayed Unions and Nonunions. A Prospective Clinical Study and Review of the Literature. Journal of Orthopaedic Surgery Research, 7, 24. http://dx.doi.org/10.1186/1749-799X-7-24

[25] Robiony, M., Polini, F., Costa, F. and Politi, M. (2002) Osteogenesis Distraction and Platelet-Rich Plasma for Bone Restoration of Severely Atrophic Mandible: Preliminary Results. Journal of Oral and Maxillofacial Surgery, 6, 630635. http://dx.doi.org/10.1053/joms.2002.33107

[26] Kitoh, H., Kitakoji, T., Tsuchiya, H., Mitsuyama, H., Nakamura, H., Katoh, M. and Ishiguro, N. (2004) Transplantation of Marrow-Derived Mesenchymal Stem Cells and Platelet-Rich Plasma During Distraction Osteogenesis-A Preliminary Result of Three Cases. Bone, 35, 892-898. http://dx.doi.org/10.1016/j.bone.2004.06.013

[27] Yoshikawa, T., Nakajima, Y., Takakura, Y. and Nonomura, A. (2005) Osteogenesis with Cryopreserved Marrow Mesenchymal Cells. Tissue Engineering, 11, 152-160. http://dx.doi.org/10.1089/ten.2005.11.152 